\title{
One-step production of pyrene-1-boronic acid functionalized graphene for dopamine detection
}

\begin{abstract}
A facile molecular wedging method is used to exfoliate graphite flakes into graphene sheets, with concurrent functionalization to form pyrene-1-boronic acid functionalized graphene (PBA/G). Different techniques are used to characterize the prepared materials such as field emission scanning electron microscope, energy dispersive X-ray analyzer, Raman, Fourier transformed infrared spectroscopy and fluorescence spectroscopy to evaluate their structural and morphological characteristics. The intercalation of PBA into graphite sheets, followed by exfoliation can be observed under the electron microscope. Elemental analyses show that the PBA acts more than intercalant, it is functionalized onto the graphene sheets upon exfoliation to form PBA/G. Raman analysis indicates PBA/G has a lower number of graphene layers as a result of successful exfoliation by PBA. Electrochemical impedance studies show that the $\mathrm{PBA} / \mathrm{G}$ possesses high affinity for dopamine through the diol groups interaction, which renders it to have enhanced detection for dopamine.
\end{abstract}

Keyword: Functionalized graphene; Sensor; Dopamine; Pyrene-1-boronic acid; Electrochemical impedance 